\title{
Advances in rheology and flow assurance studies of waxy crude
}

\author{
Zhang Jinjun*, Yu Bo, Li Hongying and Huang Qiyu \\ National Engineering Laboratory for Pipeline Safety / Beijing Key Laboratory of Urban Oil and Gas Distribution \\ Technology, China University of Petroleum, Beijing 102249, China
}

(C) China University of Petroleum (Beijing) and Springer-Verlag Berlin Heidelberg 2013

\begin{abstract}
Flow assurance is one of the core issues in safe and economical operation of waxy crude pipelines. Its essence lies in flow and heat transfer of the crude. In the past 10 years, the authors' team has achieved a lot of innovative results in aspects of waxy crude rheology, flow assurance assessment, and pipelining technologies on the basis of decades of studies. The rheological characteristics of waxy crude are much better understood, and a method for quantitatively simulating the effect of flow shear was developed based on some theoretical breakthroughs. Studies of the mechanism of waxy crude rheology have been deepened to the quantitative level. After successful development of efficient numericalalgorithms, accurate simulations have been achieved for various complex flow and heat transfer situations in waxy crude pipelining, and a reliability-based approach to flow assurance assessment has been set up. New pipelining technologies have been developed such as batching pour-point depressant- (PPD-) treated multiple-waxy-crudes, intermittent transport of waxy crudes through long-distance pipelines, and batching hot and cold crudes. By their application, a series of problems hindering safe, efficient and flexible operation of waxy crude pipelines were tackled, demonstrating that transportation technologies for waxy crude have advanced to a new and high level.
\end{abstract}

Key words: Waxy crude, pipeline transportation, flow assurance, rheology, numerical simulation

\section{Introduction}

More than $80 \%$ of crude oils produced in China are waxy crudes. Below the wax appearance temperature (WAT), a waxy crude is characterized by its poor flowability and complex rheological behavior, which result in high energyconsumption, poor operation flexibility, and severe flow assurance problems of waxy crude pipelines. Being different from other oil and gas pipelines, the most serious threat to the safe operation of waxy crude pipelines is the oil gelling and the resulted blockage of pipelines. In other words, flow assurance is the most critical concern in transporting waxy crudes.

Safe and economical operation of waxy crude pipelines has been a severe challenge worldwide. Accordingly, it has been one of the main tasks of technological development in China's petroleum storage and transportation industry. For more than 50 years since the development of the Daqing Oilfield, Chinese researchers have conducted research in this field, and have so far gained a comprehensive understanding of the complex rheological behavior of waxy crudes, and

*Corresponding author. email: zhangjj@cup.edu.cn Received July 11, 2013 have thus established a complete system for rheological characterization of crude oils. Besides, we have also achieved a deep insight into the mechanism of waxy crude rheology and its modification, and have well understood the thermal and hydraulic characteristics of waxy crude pipelines under various operation modes. On this basis, new technologies have been successfully developed and applied industrially, which have provided powerful support to safe and economical operation of about 30,000 kilometers of crude pipeline in China, and let China's crude oil pipelining technologies be positioned at the international forefront. In this process, the Department of Petroleum Storage \& Transportation Engineering in the China University of Petroleum, acting as a vital force in talent training, basic research and technological innovation in this field, has played a crucial role.

In the last 10 years, China's pipeline industry has witnessed unprecedented high-speed development. Research on crude oil rheology and pipelining technology has also made great progress, and attained a new and high level. In this review, the representative accomplishments obtained by the authors' research team during this period are systematically summarized. These cover the whole field from mechanism studies through the development and industrial application of pipelining technologies. 


\section{Rheological characteristics and their mechanism of waxy crude}

Wax is the crucial component that significantly affects the rheological behavior of crude. At temperatures above the WAT, wax is dissolved in the crude, and the waxy crude behaves as a simple Newtonian fluid. Once a waxy crude is allowed to cool to the temperatures below the WAT, wax will precipitate, agglomerate and entrap liquid oil into its structure, making the crude change to non-Newtonian fluid and show complex behavior such as thixotropy, yield stress, and viscoelasticity, which have a significant effect on the flow assurance of waxy crude pipelines. When the mass of precipitated wax is up to $2 \%-3 \%$ of the total crude, a spongylike network of wax crystals will be formed, and this causes the crude oil to lose its flowability as a whole (Li et al, 2005), namely gelation of the oil. Besides the temperature- and time-dependence, the shear and thermal history dependence makes the rheological behavior of waxy crude more complex. Rheological characteristics are the main cornerstone for safe and economical operation of crude oil pipelines.

To quantitatively characterize the rheological characteristics of waxy crude, a family of mathematic models were developed innovatively by our team, including a mechanistic model for the viscosity-temperature relationship, models for describing the thixotropic and visco-elastothixotropic behavior, correlations between flow properties and the thermal and shear history of the oil, and models for estimation of gel point/pour point and viscosity of crude blends. We also developed a method for the simulation of flow shear that takes the entropy generation due to viscous flow as a criterion, making shear simulation advance from empirical and qualitative to theoretically-guided and quantitative. In the mechanism of waxy crude rheology, fractal characterization of morphology and wax crystal structure was attained, and furthermore, correlations were developed that depict flow properties and their modification related to oil compositions and microscopic parameters representing morphology and structure of wax crystals, further deepening understandings of mechanism of wax crude rheology to a quantitative level.

\subsection{A mechanistic model for viscosity-temperature relation of waxy crudes}

Due to different forms in which the wax exists in oil, waxy crude may exhibit either Newtonian or power-law flow behavior at different temperatures above the gel point. Based on this understanding, a model was developed by applying the theory of suspension rheology ( $\mathrm{Li}$ and Zhang, 2003), which estimates the non-Newtonian viscosity as a function of temperature and precipitated wax. Viscosities at any shear rate and at any temperature above the gel point can be predicted accurately by this model, and the parameters of the rheological model may then be obtained by regressing the viscosity vs. shear rate data.

$$
\mu_{\mathrm{a}}(\dot{\gamma})=\mu_{0}\left[1-k_{0} k(\dot{\gamma}) c\right]^{-2.5}
$$

where $\mu_{0}$ is the viscosity of the liquid continuous phase; $c$ is the mass fraction of the precipitated wax particles as a proportion of the total oil mass; $k_{0} k(\dot{\gamma})$ is the factor associating with wax crystal particles and the liquid crude properties, which may be determined by regression using Eq.(1) to a flow curve at one temperature in the nonNewtonian regime.

Verification by using 4,068 viscosity data points from 33 crudes and 14 pour-point depressant- (PPD-) treated crudes with various thermal and shear histories showed that the model predicts viscosities with an absolute average deviation of $7.6 \%$. This model may accurately predict viscosities, and may also adapt well to crudes in various conditions of thermal and shear history as well as modification, such as PPD beneficiation.

\subsection{Viscoelasticity, thixotropy and its mathematical models}

The formation of a wax crystal network results in thixotropy, yield stress, and viscoelasticity. When the applied stress is less than the yield stress, the gelled waxy crude behaves as a viscoelastic solid, and its creep behavior may be described by the mechanical-analogue Jeffery model. When the applied stress reaches or exceeds the yield stress, the gelled crude will yield after creep. The creep behavior before yielding may be depicted by the Burgers model (Hou and Zhang, 2010), and when the deformation has been accumulated to a certain value, the strain of the gelled waxy crude will increase sharply by several orders of magnitude, which is a symbol of yielding. It was found that the yield strain of gelled waxy crude shows little dependence on loading conditions, while the yield stress varies significantly (Zhang et al, 2010).

For a long time, the Houska model has been used to describe the thixotropy of waxy crudes. However, it was found that the structure breakdown predicted by this model is too rapid for waxy crudes; and on the other hand the structure of waxy crystals is partially irreversible. Consequently, a new model was developed which uses two structural parameters rather than a single one, which greatly improves the accuracy of prediction (Guo et al, 2013). However, this model was thought to be too complex mathematically, and some efforts were then made to explore simpler models (Hou and Zhang, 2005; Jia and Zhang, 2012). It is important to note that all the previous thixotropic models for waxy crudes are essentially viscoplastic, and thus they cannot depict the viscoelastic behavior before yielding. This major defect inevitably reduces the accuracy of the modeling of pipeline restart. Recently, Teng and Zhang (2013a) developed a viscoelasto-thixotropic model after thorough investigation of the viscoelasto-thixotropic behavior of waxy crude. In this new model, the shear stress is considered to be a combination of an elastic stress and a viscous stress; and the evolution of the structural parameter $\lambda$ and elastic strain $\gamma_{\mathrm{e}}$ are described by separate kinetic equations. The model is expressed as follows: 


$$
\left\{\begin{array}{l}
\tau=\lambda G_{0} h\left(\gamma_{\mathrm{e}}\right) \gamma_{\mathrm{e}}+(1-\lambda) \cdot(\lambda \Delta k+k) \dot{\gamma}^{n_{1}} \\
h\left(\gamma_{\mathrm{e}}\right)=\frac{1}{1+p_{1} \cdot \gamma_{\mathrm{e}}^{p_{2}}} \\
\frac{\mathrm{d} \lambda}{\mathrm{d} t}=\frac{1}{1+\gamma^{n_{2}}}\left[a(1-\lambda)-b \lambda \phi^{m}\right] \\
\frac{\mathrm{d} \gamma_{\mathrm{e}}}{\mathrm{d} t}=\left[g_{1}-\left(1-g_{1}\right) s \dot{\lambda}\right] \dot{\gamma} \\
g_{1}=\mathrm{e}^{-\left(p_{1} \cdot \gamma^{p_{2}}\right)} \\
\gamma=\int_{0}^{t} \dot{\gamma}\left(t^{\prime}\right) \mathrm{d} t^{\prime}
\end{array}\right.
$$

where $\tau$ is the total shear stress; $G_{0}$ is the shear modulus of the completely structured material $(\lambda=1) ; k$ is the completely unstructured consistency and $\Delta k$ is the structuredependent consistency; $n_{1}$ is the power-law index, $p_{1}$ and $p_{2}$ are dimensionless parameters related to the viscoelastic property; $\gamma$ is the shear strain; $a$ is the kinetic constant for structure buildup; $b$ is the kinetic constant for shear-induced breakdown; $\phi$ is the rate of energy dissipation; $n_{2}$ and $m$ are dimensionless fitting parameters. This model depicts nicely the whole complex rheological behavior covering the viscoelastic behavior prior to yielding, yielding, and the structure breakdown after yielding, and successfully predicts the smooth transition from elastically-dominated to viscouslydominated behavior, as shown in Fig. 1.

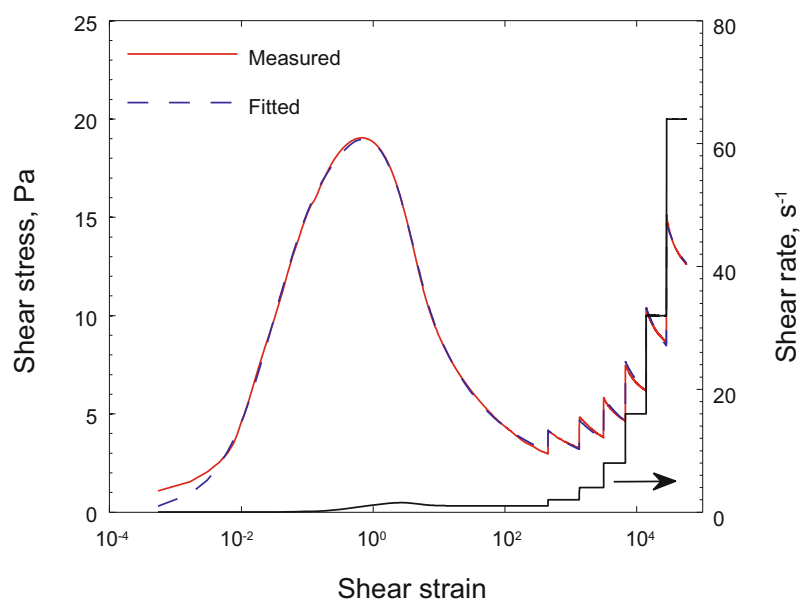

Fig. 1 Behavior in stepwise increases of shear rate and fitted results for Daqing crude at $33{ }^{\circ} \mathrm{C}$ (Teng and Zhang, 2013a)

\subsection{Correlations between rheological properties and thermal \& shear history}

Investigation into the dependence of rheological properties of waxy crude on the thermal and shear history has long remained on the level of experimental and qualitative understanding. Thanks to the findings of the role of the entropy generation due to viscous flow, correlations between the effect of shear on the gel points and viscosities of the PPD-treated crudes was established on the basis of experimental data (Li et al, 2004; Zhang et al, 2004); furthermore, an approach was explored to predict the rheological property variation of PPD-treated crudes during pipelining (Li and Zhang, 2005). In addition, based on experimental studies on Daqing crude, an approach was developed to predict the variations of viscosity with heating temperature, and correlations were obtained for the gel points and yield stress respectively as a function of flow shear and the final temperature of the dynamic cooling (Hou et al, 2009; 2010). These quantitative works not only make the rheological characteristics better understood, but also promote the application of research achievements in pipeline transportation.

\subsection{Prediction of gel point and viscosity of blended crude}

In the petroleum industry, it is a common occurrence that different crudes are blended and transported through a pipeline. However, the flow properties of blended crude do not follow the linearly-weighting rule.

Therefore, nonlinearity modification factors were introduced into the linear weighting models (Li, 1996). For the gel point, we have

$$
\left\{\begin{array}{l}
T_{\mathrm{gm}}=\sum_{i=1}^{n} X_{i} T_{\mathrm{g} i}+\sum_{j=1}^{n-1} \sum_{k=j+1}^{n}\left(B_{j k} C_{j k} X_{j} X_{k}\right) \\
B_{j k}=\left[\lg \left(100 X_{j}\right) / \lg \left(100 X_{k}\right)\right]^{\mathrm{ggn}\left(C_{j k}\right)} \\
C_{j k}=2\left(2 T_{\mathrm{g} j k}-T_{\mathrm{g} j}-T_{\mathrm{g} k}\right)
\end{array}\right.
$$

where $T_{\mathrm{gm}}$ is the gel point of blended crude; $T_{g j}$ and $T_{g k}$ are the gel points of the $j$ th and $k$ th component of crude, respectively, with $T_{\mathrm{g} j}<T_{\mathrm{g} k} ; X_{j}$ and $X_{k}$ are the mass fractions of the $j$ th and $k$ th component; $T_{g j k}$ is the gel point of the binary crude blend with equal fraction $(1: 1)$ of the $j$ th and $k$ th component, ${ }^{\circ} \mathrm{C}$; $\operatorname{sgn}\left(C_{j k}\right)$ is a sign function of $C_{j k}$, with $\operatorname{sgn}\left(C_{j k}\right)=1$ at $C_{j k}>$ 0 , and $\operatorname{sgn}\left(C_{j k}\right)=-1$ at $C_{j k}<0$. Validity and accuracy of the model were evaluated by using 259 gel points from 24 crude blends, with an absolute average deviation of $1.1^{\circ} \mathrm{C}$.

For the above model, the gel points of every binary crude blend at a mixing-ratio of 1:1 are indispensable. This makes it inapplicable when these special gel points are unavailable, e.g. when oil samples are unavailable. As a compensation, an empirical correlation of the nonlinearity modification factor was obtained by statistics of 76 gel points of binary blends with equal fraction (Chen et al, 2003). This extends the application of the nonlinearity modification model.

In estimation of the blended crude viscosity, similar nonlinearity modification factors were introduced into the Cragoe model, the Arrhenius model (the log model), and the double-log model. Of these, the modified Cragoe model was found to give the best results.

$$
\left\{\begin{array}{l}
\mu_{\mathrm{m}}=5 \times 10^{-4} \exp \left(1000 \ln 20 / L_{\mathrm{m}}\right) \\
L_{\mathrm{m}}=\sum_{i=1}^{n} X_{i} L_{i}+\sum_{j=1}^{n-1} \sum_{k=j+1}^{n} C_{j k} X_{j} X_{k} \\
L_{i}=1000 \ln 20 /\left[\ln \mu_{i}-\ln \left(5 \times 10^{-4}\right)\right] \\
C_{j k}=2\left(2 L_{j k}-L_{j}-L_{k}\right) \\
L_{j k}=1000 \ln 20 /\left[\ln \mu_{j k}-\ln \left(5 \times 10^{-4}\right)\right]
\end{array}\right.
$$


where $\mu_{\mathrm{m}}$ is the viscosity of the blended crude; $\mu_{j k}$ is the viscosity of the binary crude oil mixture with equal fraction $(1: 1)$ of the $j$ th and $k$ th component. Validity and accuracy of Eq. (4) was checked by using 3,024 sets of viscosity data of 210 blend ratios from 37 groups of crude blends, with an absolute average deviation of $9.7 \%$.

Again, in case the viscosities of every binary crude blend at a mixing-ratio of 1:1 are not available, empirical correlations of the nonlinearity modification factor $C_{j k}$ for the Arrhenius model, the double-log model and the Cragoe model were developed based on 294 viscosity data of 54 binary blends with a mixing ratio of 1:1 (Qian et al, 2006). Compared with the original Cragoe model without the nonlinearity modification, estimation using the modified model reduced the absolute average deviation from $29.5 \%$ to $19.5 \%$.

\subsection{Experimental simulation of the shear effect on waxy crude}

The rheological behavior of waxy crude is dependent on the thermal and shear history that the oil has experienced, called the thermal and shear history effect. Particularly, for PPD-treated crudes, the reduced gel point and viscosity may significantly re-rise as a result of shear (Zhang et al, 1993). Therefore, to exactly understand the effect of the major shear process in pipelining, i.e. pump shear, pipe flow shear, etc., on flow properties of PPD-treated crudes is the key point in deciding whether PPD may be used successfully or not.

To simulate the shear effect, an equation was derived to calculate the shear rate at the pipe wall for turbulent flow of a power-law fluid (Zhang et al, 1997). Furthermore, on the basis of the definition of the shear rate related to the energy dissipation rate, a set of equations were derived to determine the shear rate distribution in turbulent pipe flow for Newtonian and power-law fluids, to determine the average shear rates in flows of oil pipelining (the pipe flow, flow through a centrifugal pump and through a throttling valve), and as well to determine the average shear rate in the stirred vessel for shear simulation (Zhang, 1998). This accomplishes quantitative descriptions of all kinds of related shearing both in the oil pipelining and in the simulating apparatus.

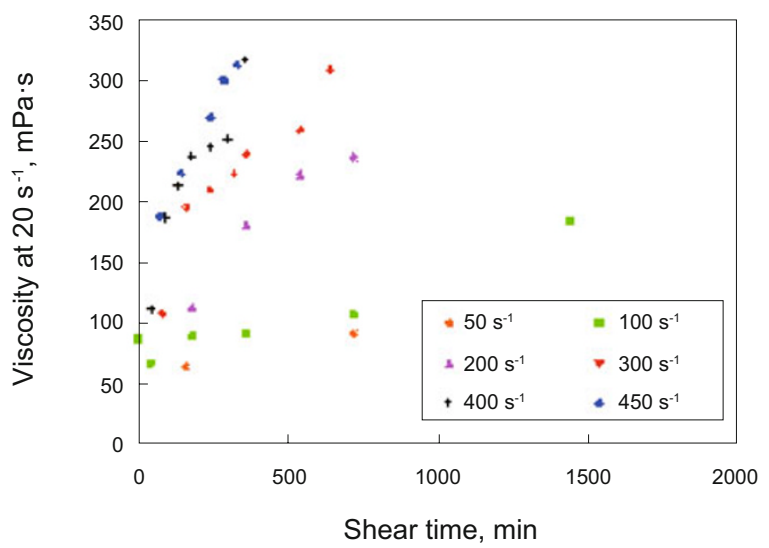

(a) Viscosity versus shear time
After careful studies, it was found that in spite of the rate and time of shear, the effects of different shear processes on the gel point and viscosity of a PPD-treated crude are the same, provided that the entropy generation due to viscous flow, or the energy dissipation, in these shear processes is the same, as shown in Fig. 2. Accordingly, we advanced that the entropy generation due to viscous flow in the shear process can be used as the scale-up criterion for simulation of the shear effect (Zhang, 1998), and thus an innovative method of quantitatively simulating the shear and thermal effects during pipelining was developed. This provides an easily-operated method for shear effect simulation, since this quantity of entropy generation both in the pipelining process and in the shear simulation apparatus can be calculated without much difficulty by means of fluid mechanics or thermodynamics. Advantages of this method also include the shortened simulation time by enhancing the shear strength, so the simulation experiment can be much more efficient. The reliability of this method has been validated by a large number of laboratory and field tests (Zhang et al, 2007). This has been recognized as a very important advance in this field.

Further studies have revealed that thixotropic behavior of waxy crude can be better depicted if the energy dissipation rate is used in the kinetic equation of thixotropic models to replace the commonly used quantities of shear rate or shear stress (Teng and Zhang, 2013a; 2013b); besides, the emulsified water content in the oil phase of a flowing oil-water system correlates well with the average energy dissipation rate in the stirred vessel. All of these indicate that the entropy generation due to viscous flow is profoundly meaningful in problems of flow.

\subsection{Mechanism of rheological behavior of waxy crude}

At temperatures below the WAT, the wax crystal morphology and structure is a key factor affecting waxy crude rheology. However, the study of rheology-microstructure relation has long stayed in a qualitative state because of the difficulties such as the high complexity and irregularity of the morphology and structure of wax crystals, the high sensitivity of the wax crystal structure to shear and thermal history, and

Fig. 2 Effect of shear at $14{ }^{\circ} \mathrm{C}$ on viscosity at $7{ }^{\circ} \mathrm{C}$ of a PPD-treated waxy crude 
also the multiple parameters of oil composition that affect the crude rheology. Breakthroughs in this frontier area have been made, and as a result Dr. Gao Peng's dissertation (Gao, 2006) was selected as the "National Excellent Doctoral Dissertation" in 2010. The main advances are as follows:

1) Morphology and structure of wax crystals were characterized, quantitatively and independently, by analysis on the microscopic images using the theory of fractal geometry and stereology (Gao et al, 2006). Fig. 3 shows wax crystal morphology and structures observed at $4{ }^{\circ} \mathrm{C}$ with a microscope, and the fractal dimension varied the measurement temperature.

2) Five representative parameters were determined by using the multivariable cluster analysis to study the correlation among the 13 factors that represent wax crystal structure and oil compositions. These five parameters are the real volume fraction of waxy crystal, the liquid phase viscosity, the average fractal dimension of wax crystals, the wax content, and the average molecular weight of crude. This makes it possible to depict the complex characteristics of crude composition and wax crystal structure with a small number of parameters.

3) Equations were developed via multivariable stepwise regression analysis, which respectively correlate the rheological parameters (viscosity, the two parameters of the power-law model, viscoelastic parameters of the low amplitude oscillation, and yield stress) with the wax crystal structure and oil composition. By means of the gray system theory, the impacts of wax crystal structure and oil composition on the gel point/pour point were illustrated quantitatively, and the role of the above-mentioned five representative parameters on rheology was sequenced.

On the basis of the above work, further studies developed a correlation between the fractal dimension increment and representative parameters of oil composition (Yi and Zhang, 2011a):

$$
\Delta D=\ln \left(9.957 \times 10^{-1} c_{\mathrm{w}}^{-0.04918} c_{\text {wax }}^{-0.02525} c_{\text {ra }}^{0.08604}\right)
$$

where $c_{\mathrm{w}}^{\prime}$ is the fraction of the precipitated wax; $c_{\text {wax }}$ is the wax content; $c_{\mathrm{ra}}$ is the content of resins and asphaltenes. This correlation, in fact, reveals the role of these three quantities in PPD modification of crude. In addition, relations that correlate shear effect and oil composition with the fractal dimension of wax crystals, gel point, viscosity, and yield stress of the PPDtreated crudes were obtained by using multiple regression analysis (Yi and Zhang, 2011b). These works indicate that the study of the mechanism of waxy crude rheology has risen to a quantitative level.

\section{Flow assurance assessment and numerical simulation of the hydraulic-thermal processes}

The flow assurance of waxy crude pipelines requires full understanding of the characteristics of the hydraulic and thermal processes in pipeline transportation. The pipeline restart failure after extended shutdown and the wax deposition are the two crucial problems involved in the flow assurance of waxy crude pipelines. In the following, advances in the numerical simulation of hydraulic-thermal processes and the reliability-based assessment of the flow assurance are illustrated in detail.

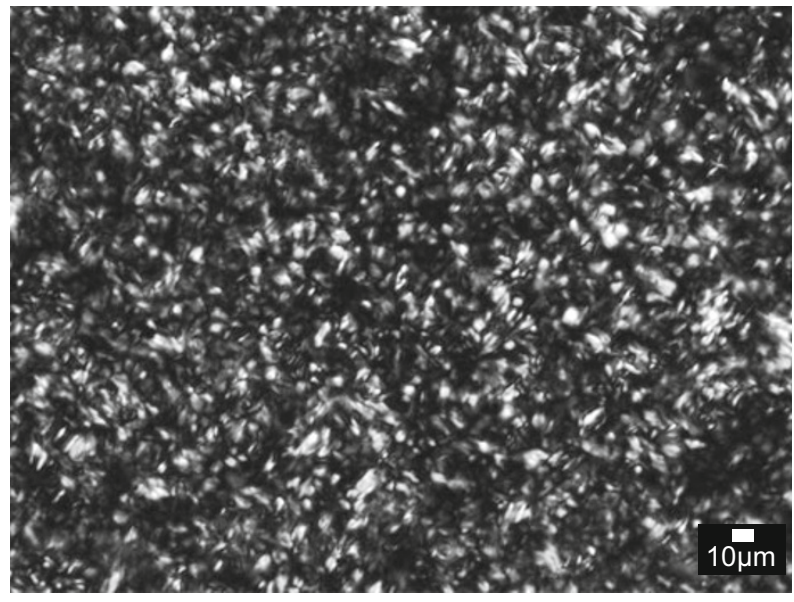

(a) Untreated

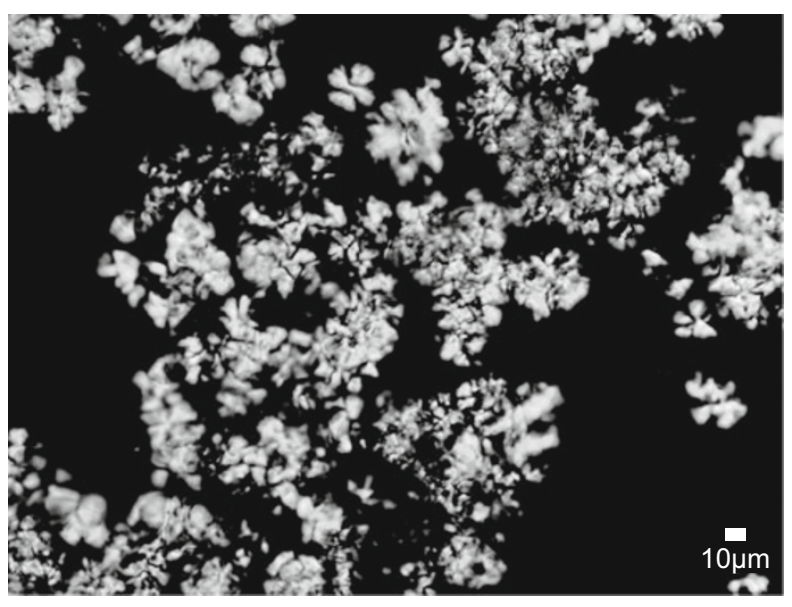

(b) Treated with a PPD

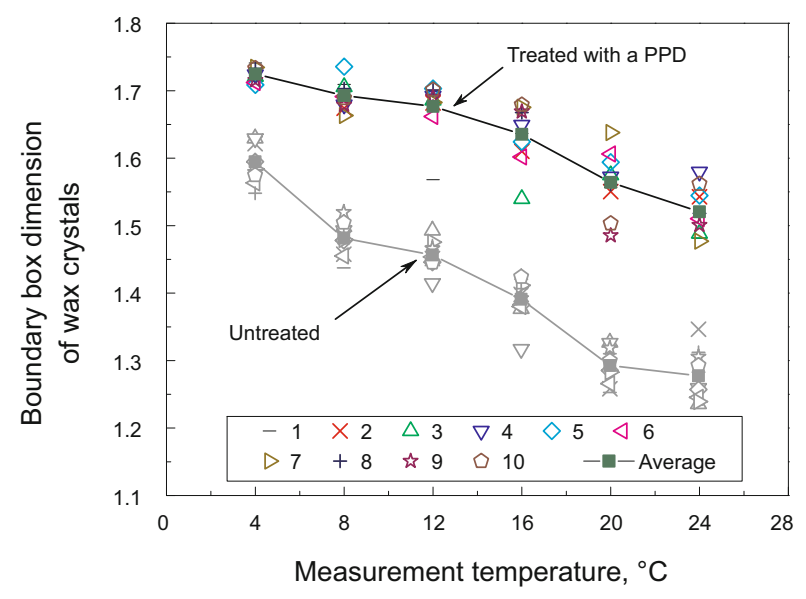

(c) Fractal dimensions versus temperature

Fig. 3 Microscopic images and fractal dimension of the wax crystal structures of a waxy crude (Gao et al, 2006) 


\subsection{Numerical simulation}

\subsubsection{Physical-mathematical model}

To solve the problems mentioned above, we proposed a more universal unsteady hydraulic-thermal-coupled mathematical model. The hydraulic model, which incorporates the thixotropic behavior of the fluid, overcomes the shortcomings of common commercial software which is not applicable for the restart computation of thixotropic crude (Yu and $\mathrm{Xu}, 2009$ ). In the thermal model, most commercial software employs an equivalent cylinder model for the soil temperature field affected by the oil pipeline. There are two drawbacks in this method: first, it is not possible to simulate accurately with such an equivalent cylinder model the strong unsteady-state processes such as the batchwise transport of cold and hot crudes or the intermittent operation of hot crude pipelines; second, the equivalent cylinder model is not applicable for dealing with the soil temperature field around two pipelines buried in one ditch. With the new model developed by our team, the computational domain of the entire thermal system can be easily obtained to overcome these two drawbacks (Yu et al, 2008).

\subsubsection{Numerical method}

The accurate and efficient prediction of thixotropic flow is a crucial procedure in hydraulic calculation of the restart process of crude pipelines. If the traditional layered hypothesis is used for the thixotropic section, different hypotheses for shear stress profile or velocity profile at different layers definitely give rise to different results. Besides, the convergence of the computation is not satisfactory and seriously time-consuming. To improve this situation, we proposed an accurate and efficient method for calculation of the restart flow, which was based on the analogy of physical meanings of different terms in the momentum equations for both Newtonian fluid and thixotropic fluid. This method achieves a unique solution by dispensing with the layered hypothesis. In addition, the time cost of this method is much less than that of the traditional layered-hypothesis method with the same grid size (Sun, 2011).

The accurate and efficient prediction of the soil temperature field is the crux in thermal calculation of the restart processes. By strict derivation, we proved that the number of triangular grids should be $4 / 3$ times larger than that of quadrilateral grids to obtain the same order of accuracy. On this occasion, the convergence rate based on quadrilateral grids is found to be faster than that based on triangular grids (Yu et al, 2012). Accordingly, we developed the calculation method based on quadrilateral grids for calculating the soil temperature field. Also, we developed an improved method to generate high-quality quadrilateral grids, with which the proportion of superior grids was increased by $20 \%$ to $60 \%$ compared with the performance of popular commercial software (Zhao et al, 2013).

\subsubsection{Hydraulic and thermal characteristics of various pipelining processes}

Based on the above improvements for mathematical modeling and numerical methods, a set of simulation software specific to waxy crude pipelining has been developed. Employing this software, we clarified the complex hydraulic- thermal characteristics in various pipeline operation situations such as the parallel-laying of two pipelines in one ditch, batchwise transport of cold and hot crudes, intermittent transport of waxy crude through long-distance pipelines, etc. In addition, our studies also uncovered some problems in existing engineering specifications and provided a theoretical basis for development of safe, energy-saving and more environmentally-friendly pipelining techniques.

To clarify the thermal impacts of two pipelines, one hot crude line and one product line buried in one ditch, we focused on the variations of oil temperature in the two pipelines under different pipeline intervals, buried depth, soil physical properties, pipeline throughput and ground temperature, etc., and revealed the thermal influence mechanism between the two pipelines. Results showed that, the product pipeline has minor impacts on the crude pipeline if the pipeline interval is greater than $1.2 \mathrm{~m}$. This work solved the problem of determining the thermally-safe pipeline interval (Yu et al, 2007; 2008).

In the study of batching cold and hot crudes, we clarified the strong unsteady-state hydraulic-thermal characteristics in this transporting process. It is found that pre-heating the cold oil tail and decreasing the temperature of the hot oil tail may reduce heating energy considerably. After optimizing the 'heating period' and 'cooling period, safe and energy-saving operation scenarios were set up (Wang et al, 2009).

For the intermittent transport of waxy crudes, the general hydraulic-thermal characteristics of this operation mode were investigated by means of numerical simulation combined with field tests. We gained a better understanding of the operation features of the intermittent transport by analysis and comparison of the transient thermal characteristics of intermittent and continuous transport (Liu et al, 2010).

\subsection{Reliability-based flow assurance assessment}

Restart failure after extended shutdown has always been a serious concern in waxy crude pipelines. Previous studies of restartability of waxy crude pipelines were conducted via a deterministic approach. However, oil properties, soil and operation parameters, which contribute to the restartability, are all subject to uncertainty. Consequently, this deterministic method cannot provide a comprehensive and scientific assessment. Therefore, we introduced the reliability-based method to solve this problem. A limit state function based on the flow rate was developed, which considers the uncertainty of variables such as operation, oil properties, soil and environmental conditions (Yu et al, 2013c).

$$
g(\boldsymbol{x})=Q_{\mathrm{s}}-Q_{\mathrm{c}}
$$

where the vector $\boldsymbol{x}$ represents the random variables related to the restart process; $Q_{\mathrm{s}}$ is the recovered flow rate at the outlet of the pipeline during the restart, which is obtained by stochastic numerical simulation; $Q_{\mathrm{c}}$ is the critical safe flow rate.

When Monte-Carlo simulation is employed to obtain the failure probability, high computational complexity and huge memory capacity are key problems. We modified the proper 
orthogonal decomposition (POD) algorithm and extended it to the thermal calculation for a crude pipeline. A POD-Galerkin reduced order model was developed for the first time for steady and unsteady hydraulic-thermal processes. By using this model to solve the steady problems, calculation may be speeded up by hundreds or even thousands of times, and even for unsteady problems calculation may be accelerated by dozens of times (Yu et al, 2013a; 2013b).

By using this assessment method, restart failure probability was studied as a function of variables such as the pumping temperature, flow rate, pipe diameter, soil temperature, etc. (Yu et al, 2013c). Fig. 4 shows the restart failure probability varied with the inlet temperature calculated for a pipeline. And furthermore, approaches were set up for determination of pumping temperature and allowable time of pipeline shutdown. Compared to the conventional approach that determines the pumping temperature based on crude pour point, the reliability-based method gives results quantitatively and precisely. This, both in theory and methodology, provides powerful support to the safe and economical operation of waxy crude pipelines.

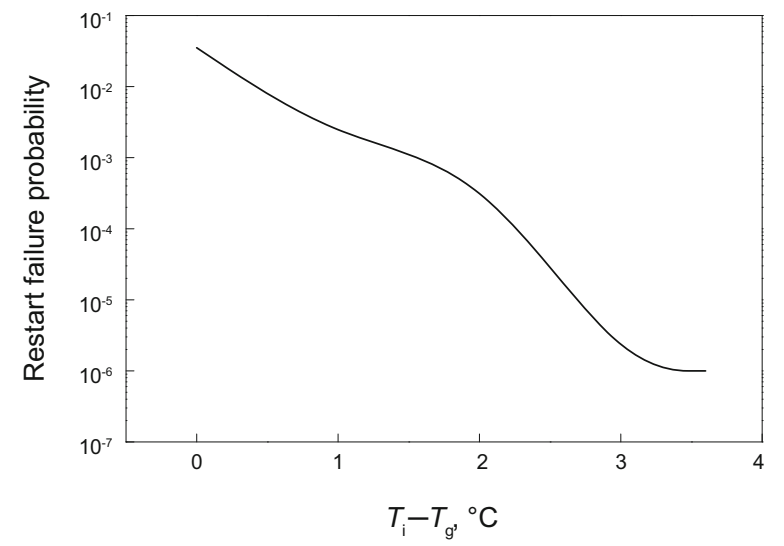

Fig. 4 Restart failure probability varied with the difference between the inlet oil temperature $\left(T_{\mathrm{i}}\right)$ of the station and the gel point $\left(T_{\mathrm{g}}\right)$

\subsection{Wax deposition}

Wax deposition is an important factor that affects safe and economical operation of waxy crude pipeline. The mechanism and prediction of wax deposition is a topical issue in flow assurance studies.

A kinetic model of wax deposition was developed based on a molecular diffusion mechanism and experimental results in a flow loop (Huang et al, 2006).

$$
W=k \tau_{\mathrm{w}}^{m} \frac{1}{\mu}\left(\frac{\mathrm{d} C}{\mathrm{~d} T}\right)\left(\frac{\mathrm{d} T}{\mathrm{~d} r}\right)^{1+n}
$$

where $W$ is the wax deposition rate; $\tau_{\mathrm{w}}$ is the shear stress at the pipe wall; $\mathrm{d} C / \mathrm{d} T$ is the amount of precipitated wax at every $1{ }^{\circ} \mathrm{C}$ of cooling, i.e. the solubility coefficient of wax in crude oil; $\mathrm{d} T / \mathrm{d} r$ is the radial temperature gradient at the pipe wall; $k, m$, and $n$ are fitting parameters.

This model takes into consideration the impact of pipe flow shear. One of its disadvantages is that the model parameters have to be determined with a large number of experimental data from flow loop simulation. Later, a unified model was developed after studies of flow and wax precipitation within a pipe (Huang et al, 2008). With this unified model, the wax deposition profile along a pipeline may be predicted using only limited oil properties, such as oil viscosity and the wax precipitation curve.

So far, these models have been used to predict the wax deposition in a couple of domestic and overseas pipelines, providing a sound basis for pigging.

\section{Development and application of safe and efficient pipeline transportation technologies}

Traditionally, waxy crudes are transported with heating. China's operation technical code of crude pipelines requires that the inlet temperature at the heating station or the terminal of long-distance pipelines should be controlled at $3{ }^{\circ} \mathrm{C}$ above the gel point. Heating may not only decreases the viscosity so as to reduce hydraulic loss, but also reduces the risk of pipeline restart failure after extended shutdown. For buried hot oil pipelines at low throughput rates, the operation optimization studies revealed that the optimal inlet temperature is the lowest allowable temperature required by flow assurance, and it will increase when the throughput is close to the designed capacity. Restart failure and wax deposition, the two major concerns of flow assurance, can be solved effectively by controlling shutdown time and regular pigging respectively.

Operation of $30,000 \mathrm{~km}$ of crude pipeline in China has demonstrated that this heating technique and the corresponding flow assurance measures are effective. As an example, the Northeast Pipeline System (total length 2,300 $\mathrm{km}$, O.D. $720 \mathrm{~mm}$ ) transporting the Daqing crude, which has a gel point of $32{ }^{\circ} \mathrm{C}$ or a pour point of $35^{\circ} \mathrm{C}$, has operated safely for more than 40 years by using this technique. The shortcomings of the heating technique lie in the high energyconsumption, short allowable time of shutdown (for the Northeast Pipeline System, this time is $20 \mathrm{~h}$ in winter and 30 $\mathrm{h}$ in summer), and poor operation flexibility, i.e. the flow rate of the pipeline cannot be too low.

To transport waxy crude more safely and more economically, other techniques were developed such as water suspension, heat treatment/thermal conditioning, and more recently PPD-modification. In recent years, our team successfully developed some new methods for transporting waxy crude through long-distance pipelines and applied them to the newly constructed China West Crude Pipeline, including batching of multiple waxy-crudes treated with PPD, intermittent transport of waxy crude, and batching of hot and cold crude. This advances waxy crude pipelining technology to a new level.

\subsection{Heat treatment and PPD-modification}

The heat treatment is to heat waxy crude to a high temperature, and then cool it down under proper rate and shear conditions. This may reduce the gel point and viscosity of waxy crude. In the $1980 \mathrm{~s}$, the Ma-Hui-Ning pipeline $(270 \mathrm{~km})$ and the $\mathrm{Pu}$-Lin pipeline $(242 \mathrm{~km})$ adopted this 
technology to tackle the problem of low throughput operation.

By adding proper PPD, wax crystal morphology and structure can be improved greatly, leading to significant reduction in the gel point and non-Newtonian viscosity, and hence the pipeline can be operated more safely, more economically and more flexibly. This technology began to be widely used in China in the 1990s. More than 20 pipelines have now used this technology beneficially, in which a typical example is the Lu-Ning Pipeline (Qian et al, 2004).

The Lu-Ning Pipeline, $665 \mathrm{~km}$ long and $720 \mathrm{~mm}$ O.D., was designed to transport $2,000 \times 10^{4} \mathrm{t} / \mathrm{a}$ of crude via heating. There are an initial and a terminal station, as well as 11 intermediate pump and heating stations. At the early 1990s, consumption of heating fuel rose significantly due to declined throughput and poor flowability of the crude. By adding $50 \mathrm{~g} /$ t PPD and heating the oil with PPD up to $60{ }^{\circ} \mathrm{C}$, the gel point and viscosity at $25^{\circ} \mathrm{C}$ at the initial station was reduced by 18 ${ }^{\circ} \mathrm{C}$ and $84 \%$, respectively. This reduced the inlet temperature at the heating station from $34{ }^{\circ} \mathrm{C}$ to $27 \pm 1{ }^{\circ} \mathrm{C}$, and the heating station operation mode changed greatly from all 12 stations being run for the whole year to only one station being run in summer, 2-5 stations run in spring and autumn, and every second in winter. This greatly reduced heating fuel use by $17,200 \mathrm{t}$ in a year.

Flow properties of PPD-treated crudes depend strongly on the shear and thermal history. In the early years of PPD application, it was mainly used in existing pipelines designed and operated by adopting the heating method, and the heating stations stopped operation one after another after the effectiveness of the PPD was validated through field tests. However, with PPD being extended to use in newly constructed pipelines, the effectiveness of PPD and flow property variation during pipelining has to be accurately predicted in the pipeline design stage. The novel simulation method mentioned above, which is based on the entropy generation due to viscous flow, solves this bottle-neck problem of PPD application.

\subsection{Development and application of new technologies}

In the last decade, China's imported petroleum sources have been diversified, and the quality of domestically produced crude has been worsening. Therefore, transporting multiple crudes through a single pipeline becomes a common occurrence, and properties of the transported crudes differ from each other more and more greatly. Conventionally, the mixing of different crudes was often conducted when multiple crudes with waxy or heavy crudes among them were required to be transported in a pipeline for the sake of flow assurance. However, mixing of different crudes is not beneficial to trade and processing of crudes, particularly for those oils with good quality. The China West Crude Pipeline (CWCP), a strategic energy-transportation channel in western China, is a typical example that multiple crudes with considerably different properties have to be transported in a single pipeline.

The CWCP, 1,838 km long, starts from Urumqi, via Shanshan and Yumen, and ends at Lanzhou. It consists of the trunk line from Shanshan to Lanzhou $(1,541.2 \mathrm{~km}$ long and $813 / 711 \mathrm{~mm}$ O.D.) and the Urumqi-Shanshan branch line
(296.5 $\mathrm{km}$ long and $610 \mathrm{~mm}$ O.D.). The designed pressure of the system is $8.0 \mathrm{MPa}$, and the capacity is $2,000 \times 10^{4} \mathrm{t} /$ a for the trunk line and $1,000 \times 10^{4} \mathrm{t} / \mathrm{a}$ for the branch line. There are 9 intermediate pump stations along the trunk line. Construction of the pipeline was begun in August 2004, and completed in August 2007.

The crudes transported through the CWCP include three crudes produced in northwest China's Tarim Basin, the Tuha Basin and the north Xinjiang oilfield, and one crude imported through the Kazakhstan-China Oil Pipeline. The oil properties of the multiple crudes, not only differ considerably from each other, but also vary greatly in one crude. Take the gel point as an example, it varies from -11 to $-3{ }^{\circ} \mathrm{C}$ for the Tarim crude, and varies from 2 to $14{ }^{\circ} \mathrm{C}$ for the north Xinjiang crude; and varies from -4 to $19{ }^{\circ} \mathrm{C}$ for the Tuha crude; and varies from -12 to $22{ }^{\circ} \mathrm{C}$ for the Kazakhstan crude. Design and operation of the CWCP faced severe challenges in flow assurance as a combined result of following reasons, i.e. low environment temperature (the lowest soil temperature at the buried depth is below $2{ }^{\circ} \mathrm{C}$ ), highly variable transporting requirement, complicated thermal interaction between the crude line and the product line buried $1.2 \mathrm{~m}$ apart from the crude line.

Thanks to the advances in waxy crude rheology and flow assurance studies, these problems were tackled effectively, and new technologies for multiple waxy-crude transport were developed and used in design and operation of the CWCP, ensuring its on-time commissioning and safe and efficient operation. The main technological advances are as follows.

Batching multiple crudes treated with PPD (Ling et al, 2008) Based on the quantitative simulation of the shear and thermal effect during pipelining and flow assurance assessment, the PPD-modification was promoted to batching multiple waxy crudes through long distance pipelines, that is, conducted proper PPD treatment according to the crude properties. The use of this method successfully solved the problems of both the thermal constraint resulted from big spacing between two adjacent stations and low throughput operation. In winter, the crudes were treated with PPD at Urumqi and Shanshan stations (the crudes PPD-treated at Urumqi will not be re-treated at Shanshan), and the PPDtreated crudes can be transported either to Yumen (the Tuha crude) or Lanzhou (other crudes) without successive heating. The pipeline ran more safely and economically, with fuel consumption reduced by $83 \%$ compared with the heating method.

Intermittent transport for long-distance waxy crude pipelines (Liu et al, 2010) The difficulty of intermittently transporting waxy crude lies in that the possibility of restart failure could be much higher than in continuously operated pipelines. This is mainly attributed to the lower thermal storage in the soil and the resultant rapid cooling of the oil. Besides, the difficulty of intermittent operation simulation comes from the endlessly varying soil temperature, which is also a function of the pipeline operation history. To tackle the problems from intermittent operation of the CWCP, on one side PPD was used to improve the intrinsic safety of flow, and on the other side numerical simulation was performed to understand the hydraulic and thermal characteristics of the 
intermittent operation, and further to optimize the operation scenarios. Field tests were conducted to validate the reliability of this technique, and finally intermittent transport achieved success in the CWCP. When full delivery of the Tuha crude at the Yumen station is conducted, the downstream YumenLanzhou segment, $792 \mathrm{~km}$ long, is operated in an intermittent manner, with averagely shut down for 1 day after 5.4 days of running and $25.4 \mathrm{~h}$ of mean time for shutdown. The intermittent operation was conducted from January to April 2009 , when the CWCP was impacted by the global financial crisis and the throughput was reduced by a quarter of the designed level.

Batching hot and cold oil (Wang et al, 2009) Nearly $50 \%$ of the crudes transported through the CWCP can be pumped without heating all year; and the remaining crudes have to be heated to $55^{\circ} \mathrm{C}$ at the initial station in winter to achieve PPD-modification. Heat consumption may be greatly reduced if the crudes having good flowability are pumped without heating. However, this way of transporting hot and cold oil batchwise is difficult because the oil temperature, pressure, and soil temperature result in a complex alternating process, and besides the hydraulic and thermal conditions interact with each other. Therefore, flow assurance is much more difficult than for the usual hot crude pipelines. Moreover, the structural safety of pipelines under alternating loads is also a critical concern. At that time, this way of oil pipeline usage was only reported used in the Pacific Pipeline System in California, U.S., which is $209 \mathrm{~km}$ long, 508 $\mathrm{mm}$ O.D., and was commissioned in 1999. Via numerical simulations on this transport process, we understood well the hydraulic and thermal characteristics, and further optimized the transport scenario. The safety and reliability were confirmed after the analysis on the strength, stability and fatigue life of the pipeline structure, and restartability after extended shutdown. This technology was finally put into use after field tests. Now it has become one of the regular operation modes of the CWCP. Each year in the early winter from November and December and in the early spring from March and April, Tuha crude is transported using PPDtreatment, while the remaining crudes are transported without heating.

According to various transportation requirements and by the integrated use of these new technologies, the CWCP has successfully dealt with a variety of transport challenges and met the demands from both the oil fields and the downstream petrochemical enterprises. The pipeline therefore has attained safe, efficient and flexible operation.

\section{Concluding remarks}

Besides the decades of unremitting endeavor from generations of researchers in our university, these achievements also rely on the close combination between research and engineering application, and the ever-deepening basic research. We have not only achieved a lot in basic studies such as rheology mechanisms, experimental method, and numerical methods, we have also been actively involved in resolving engineering problems of many important pipelines in China and abroad. Indeed, some excellent studies have also been conducted in this field in foreign universities, but most of those studies were mainly focused on mechanistic problems, somewhat removed from actual engineering practice, and the applied technologies are mainly studied by company R\&D institutions. On the whole, company R\&D institutions, domestic and overseas, cannot compare with the universities in the basic research of this field. Though previously we lagged behind foreign universities in some of the basic studies because of restrictions in instruments and awareness etc., things have changed a lot in the past ten years. This can be inferred more or less from our published works in international journals. In the future, we should still stick to the strategy of conducting research in the entire front from the basic investigations to engineering applications.

With ever-decreasing availability of conventional oils, the output of waxy crude throughout the world has increased significantly since the 1990s. The rheology and pipeline transportation of waxy crude has become quite an active research field. Future studies will focus on the rheological behavior of single and multi-phase waxy crude systems as well as flow improvement mechanisms and technologies, depositions and their mechanisms of wax and asphaltene etc., flow assurance of the single- and multi-phase pipelines of waxy crude in various complex flow conditions, etc. These will also be our future tasks.

\section{Acknowledgements}

We are grateful to the strong and long-term support from the National Natural Science Foundation of China (Grant Nos. 51134006, 50944030), the Ministry of Education (Grant No. 104118), Beijing Municipal Education Commission (Grant No. YB20081141401), and companies such as China National Petroleum Corporation (CNPC), China Petrochemical Corporation (SINOPEC).

\section{References}

Chen J, Zhang J J and Zhang F. New approach developed for estimating pour point of crude oil blends. Oil \& Gas Journal. 2003. 101(31): 60-64

Gao P. Study on Relation of Waxy Crude Rheology to Its Composition and Wax Crystal Morphology and Structure. Ph.D. Thesis. China University of Petroleum (Beijing). 2006 (in Chinese)

Gao P, Zhang J J and Ma G X. Direct image-based fractal characterization of morphologies and structures of wax crystals in waxy crude oils. Journal of Physics: Condensed Matter. 2006. 18(50): 11487-11506

Guo L P, Zhang J J, Han S P, et al. Evaluation of thixotropic models for waxy crudes based on stepwise shearing measurements. Petroleum Science and Technology. 2013. 31(9): 895-901

Hou L and Zhang J J. Study on thixotropy of waxy crude based on viscoelasticity analysis. Journal of China University of Petroleum (Edition of Natural Science). 2005. 29(4): 84-86 (in Chinese)

Hou L and Zhang J J. A study on creep behavior of gelled Daqing crude oil. Petroleum Science and Technology. 2010. 28(7): 690-699

Hou L, Zhang J J, Ding J L, et al. The variation of the gel point of Daqing crude oil with pipelining history. Petroleum Science and Technology. 2010. 28(13): 1331-1337

Hou L, Zhang J J and Sun L. Change of yield stress of Daqing crude oil with thermal and shear history. Petroleum Science and Technology. 
2009. 27(18): 2168-2176

Huang Q Y, Li Y X and Zhang J J. Unified wax deposition model. Acta Petrolei Sinica. 2008. 29(3): 459-462 (in Chinese)

Huang Q Y, Zhang J J, Gao X F, et al. Study of wax deposition from Daqing crude oil. Acta Petrolei Sinica. 2006. 27(4): 125-129 (in Chinese)

Jia B L and Zhang J J. A six-parameter thixotropic model for waxy crude oil. Acta Petrolei Sinica. 2012. 33(6): 1080-1085 (in Chinese)

Li C W. Rheological Properties and Their Correlations of Crude Oil Blends. M.S. Thesis. China University of Petroleum (Beijing). 1996 (in Chinese)

Li H Y and Zhang J J. A generalized model for predicting nonNewtonian viscosity of waxy crudes as a function of temperature and precipitated wax. Fuel. 2003. 82(11): 1387-1397

Li H Y, Zhang J J and Yan D F. Correlations between the pour point/gel point and the amount of precipitated wax for waxy crudes. Petroleum Science and Technology. 2005. 23(11-12): 1313-1322

Li Y F and Zhang J J. Prediction of viscosity variation for waxy crude oils beneficiated by pour-point-depressants during pipeline. Petroleum Science and Technology. 2005. 23: 7-8

Li Y F, Zhang J J and Hang Q Y. A mathematical model for shear effect on the viscosity of waxy crude beneficiated with pour-point depressants. Acta Petrolei Sinica. 2004. 25(4): 109-112 (in Chinese)

Ling X, Zhang J J, Li H Y, et al. Transportation of waxy crudes in batch through China west crude oil pipeline with pour-point-depressant beneficiation. Proceedings of the ASME 7th International Pipeline Conference 29 September -3 October 2008, Calgary, Canada

Liu X, Zhang J J, Li H Y, et al. Intermittent operations, PPD optimize low flow-rate waxy crude system. Oil \& Gas Journal. 2010. 108(46): 134-138

Qian J H, Zhang J J, Li H Y, et al. Study evaluates viscosity prediction of crude blends. Oil \& Gas Journal. 2006. 104(39): 61-68

Qian J H, Zhang J J, Wang K Z, et al. Pipelining of viscous waxy crude with the pour-point-depressant beneficiation: successful experience in the Lu-Ning pipeline. Proceedings of the ASME 5th International Pipeline Conference, 4-8 October 2004, Calgary, Alberta, Canada

Sun C Z. Numerical study on the shutdown and restart of a subsea pipeline. Ph.D. Thesis. China University of Petroleum. 2011 (in Chinese)

Teng H X and Zhang J J. Modeling the thixotropic behavior of waxy crude. Industrial \& Engineering Chemistry Research. 2013a. 52(23): 8079-8089

Teng H X and Zhang J J. Modeling the viscoelasto-plastic behavior of waxy crude. Petroleum Science. 2013b. 10(3): 395-401

Wang K, Zhang J J, Yu B, et al. Numerical simulation on the thermal and hydraulic behaviors of batch pipelining crude oils with different inlet temperatures. Oil \& Gas Science and Technology. 2009. 64(4): 503-520

Yi S Z and Zhang J J. Relationship between waxy crude oil composition and change in the morphology and structure of wax crystals induced by pour-point-depressant beneficiation. Energy \& Fuels. 2011a. 25(4): 1686-1696

Yi S Z and Zhang J J. Shear-induced change in morphology of wax crystals and flow properties of waxy crudes modified with the pourpoint depressant. Energy \& Fuels. 2011b. 25(12): 5660-5671

Yu B, Ling X, Zhang J J, et al. Study of laying technology of a products pipeline along with a hot crude pipeline in one ditch. Acta Petrolei Sinica. 2007. 28(5): 149-152 (in Chinese)

Yu B, Wang Y, Zhang J J, et al. Thermal impact of the products pipeline on the crude oil pipeline laid in one ditch: the effect of pipeline interval. International Journal of Heat and Mass Transfer. 2008. 51(3): 597-609

Yu B and $\mathrm{Xu} \mathrm{C}$. Study of restart of crude oils batch pipelined with different outlet temperatures. Oil \& Gas Storage and Transportation. 2009. 28: 4-16 (in Chinese)

Yu B, Yu G J, Cao Z Z, et al. Fast calculation of the soil temperature field around a buried oil pipeline using a body-fitted coordinatesbased POD-Galerkin reduced-order model. Numerical Heat Transfer, Part A: Applications. 2013a. 63(10): 776-794

Yu G J, Yu B, Han D X, et al. Unsteady-state thermal calculation of buried oil pipeline using a proper orthogonal decomposition reducedorder model. Applied Thermal Engineering. 2013b. 51 (1-2): 177-189

Yu G J, Yu B, Zhao Y, et al. Comparative studies on accuracy and convergence rate between the cell-centered scheme and the cellvertex scheme for triangular grids. International Journal of Heat and Mass Transfer. 2012. 55(25-26): 8051-8060

Yu P F, Zhang J J and Yu B. Efficient stochastic numerical simulation methods for restart failure probability calculation of a waxy crude pipeline. The 4th Asian Symposium on Computational Heat Transfer and Fluid Flow, 3-6 June 2013c, Hong Kong

Zhang J J. Shear Effect in Pipelining Waxy Crude Oils Treated with the Pour-point Depressant and Its Simulation. Ph.D. Thesis. China University of Petroleum (Beijing). 1998 (in Chinese)

Zhang J J, Liu Z H, Zhang F, et al. Waxy crude treated with pour-pointdepressants: flow behavior and its evaluation. Proceedings of the 1997 International Symposium on Multiphase Fluid, 7-11 October 1997, Beijing, China. 715-720

Zhang J J, Pan D L, Tu H M, et al. A mathematical model for the shear effect on the gel point of crude beneficiated with pour-point depressants. Acta Petrolei Sinica. 2004. 25(2): 96-99 (in Chinese)

Zhang J J, Peng J W and Han S P. Yielding behaviors of gelled waxy crude oil on different stress loading conditions. Proceedings of the 5th Pacific Rim Conference on Rheology, 1-6 August 2010, Sapporo, Japan

Zhang J J, Zhang F, Huang Q Y, et al. Experimental simulation of effect of shear on rheological properties of beneficiated waxy crude oils. Journal of Central South University of Technology. 2007. 14: 108111

Zhang J J, Zhao T H, Liu Z H, et al. Effect of shear on rheology of Xinjiang crude oil treated by flow improver. Journal of China University of Petroleum (Edition of Natural Science). 1993. 17(6): 74-78 (in Chinese)

Zhao Y, Yu B and Tao W Q. An improved paving method of automatic quadrilateral mesh generation. Numerical Heat Transfer, Part B: Fundamentals. 2013. 64(3): 218-238

(Edited by Sun Yanhua) 